\title{
Near-equatorial Pi2 and Pc3 waves observed by CHAMP and on SAMBA/MAGDAS stations
} \author{
A. Yoshikawa ${ }^{\mathrm{f}}$ \\ ${ }^{\text {a }}$ Serena University, La Serena, Chile \\ ${ }^{\mathrm{b}}$ Space Research Institute, Moscow, Russia \\ ${ }^{\mathrm{c}}$ Geological and Geophysical Institute of Hungary, Tihany, Hungary \\ ${ }^{\mathrm{d}}$ University of Santiago de Chile, Santiago, Chile \\ ${ }^{\mathrm{e}}$ GFZ German Research Centre for Geosciences, Potsdam, Germany \\ ${ }^{\mathrm{f}}$ Kyushu University, Fukuoka, Japan
}

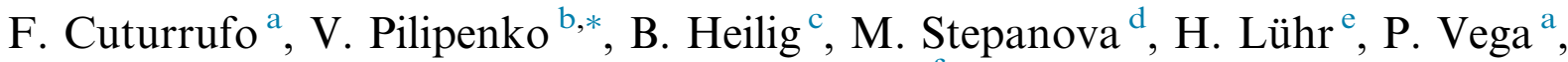

Received 7 January 2014; received in revised form 20 October 2014; accepted 12 November 2014

\begin{abstract}
We have examined simultaneous ULF activity in the Pi2 and Pc3 bands at the near-equatorial magnetic stations in South America from SAMBA and MAGDAS arrays and low-orbiting CHAMP satellite during its passage over this meridional network. At the nighttime, both Pi2 and Pc3 waves in the upper ionosphere and on the ground are nearly of the same magnitude and in-phase. At the same time, the daytime Pc3 pulsations on the ground and in space are nearly out-of-phase. Comparison of observational results with the theoretical notions on the MHD wave interaction with the system ionosphere-atmosphere-ground suggests that nighttime low-latitude Pi2 and Pc3 wave signatures are produced by magnetospheric fast compressional mode. The daytime near-equatorial Pc3 waves still resist a quantative interpretation. These waves may be produced by a combination of two mechanisms: compressional mode leakage through the ionosphere, and by oscillatory ionospheric current spreading towards equatorial latitudes.
\end{abstract}

(C) 2014 COSPAR. Published by Elsevier Ltd. All rights reserved.

Keywords: ULF waves; Ground magnetometers; CHAMP satellite; Geomagnetic equator

\section{Introduction: possible mechanisms of $\mathrm{Pi} 2 / \mathrm{Pc} 3$ wave propagation to low latitudes}

The physics of ultra-low-frequency (ULF) waves in the magnetosphere did not pay much attention to MHD fast mode. It was assumed that this isotropically propagating mode, when excited in the outer magnetosphere, would

\footnotetext{
* Corresponding author.

E-mail addresses: fcuturrufo@gmail.com (F. Cuturrufo), space.soliton@gmail.com (V. Pilipenko), heilig@elgi.hu (B. Heilig), marina. stepanova@usach.cl (M. Stepanova), hermann.luehr@gfz-potsdam.de (H. Lühr), vegapedro@gmail.com (P. Vega),yoshi@geo.kyushu-u.ac.jp (A. Yoshikawa).
}

spread over entire terrestrial space environment, so just a very small part of its wave energy could reach our relatively small planet. Therefore, all efforts were concentrated basically on the examination of Alfven MHD waves, which can transport wave energy along field lines to large distances without any geometrical attenuation. Lately, this paradigm has started to change in view of recent observational results on $\mathrm{Pi} 2$ and $\mathrm{Pc} 3$ pulsations.

Nighttime Pi2 pulsations (typical time scale from fraction to few $\mathrm{min}$ ) are impulsive, damped geomagnetic field oscillations, which are best known for their occurrence at the time of magnetospheric substorm (e.g. Keiling and Takahashi (2011)). Impulsive or quasi-periodic intensifications in the 
magnetotail during non-substorm periods also can induce Pi2 pulsations (Kepko and Kivelson, 1999; Sutcliffe and Lyons, 2002; Kim et al., 2005). At high latitudes, Pi2 pulsations are typically observed around the local midnight and are associated with the substorm onset. Although auroral substorm disturbance is confined to a relatively narrow range of longitudes around local midnight, low-latitude Pi2s occur over an extremely large range of longitudes (Yumoto et al., 1990; Li et al., 1998). These results have suggested a global cavity mode as a mechanism of low-latitude Pi2 pulsations (Yeoman and Orr, 1989).

The cavity mode concept was proved by simultaneous detection of Pi2 disturbances on satellites and on the ground. Direct comparison (Takahashi et al., 1995) of nightside Pi2 at AMPTE satellite and low-latitude ground station showed a high coherence between compressional (field-aligned) component in space and $\mathrm{H}$-component on the ground when satellite was inside the plasmasphere $(2<L<4)$. Multisatellite and ground studies of the amplitude-phase radial structure of Pi2 pulsation by Teramoto et al. (2008) further confirmed the concept of the cavity mode excitation in the inner magnetosphere $(L<5)$. A comparative study of $\mathrm{Pi} 2$ pulsations observed by the low-altitude CHAMP satellite and at low-latitude ground station (Sutcliffe and Lühr, 2003) indicated that the H-component of the $\mathrm{Pi} 2$ pulsation on the ground is well correlated with the compressional and poloidal components in the upper ionosphere. Han et al. (2004) also found that the field-aligned component of Pi2 pulsations at the low-orbiting Ørsted satellite is correlated with the $\mathrm{H}$ component on the low-latitude magnetic observatories. According to the cavity mode concept, frequency of Pi2 pulsations is determined by eigenfrequency of the inner-magnetospheric cavity mode. Such mode is leaky one, so weak Pi2 signatures were detected by a loworbit satellite even in the polar cap (Teramoto et al., 2011).

Intriguing peculiarity of low-latitude ground $\mathrm{Pi} 2$ is that they are often observed on the dayside at near-equatorial latitudes simultaneously with their intense nighttime counterparts (Sutcliffe and Yumoto, 1991) with negligible time delay between them (Nosé et al., 2006). These observations may evidence a global scale of Pi2 cavity mode. However, Takahashi et al. (1995) could not find evidence for dayside Pi2 pulsations in AMPTE-CCE satellite data at low latitudes within the plasmasphere. The search for daytime Pi2 pulsations in CHAMP satellite data at low orbit was also unsuccessful (Sutcliffe and Lühr, 2010). Thus, there is no indication that $\mathrm{Pi} 2$ pulsations propagate to the dayside through the upper ionosphere or magnetosphere. Moreover, Han et al. (2004) presented two dayside cases where they observed oscillations in Pi2 band on Ørsted and found that these oscillations were significantly smaller and out-of-phase with those observed on the ground. They concluded that dayside Pi2s are not due to a global cavity resonance, but are more likely caused by a current flowing in the $\mathrm{E}$ region ionosphere along the equator.

Wave energy transport from dayside outer magnetosphere to the ground by fast compressional mode is important for Pc3 waves as well (Takahashi et al., 1994; Kim and Takahashi, 1999). The coordinated low-altitude satellite (CHAMP) and ground observations of Pc3-4 waves indicated on a significant contribution of compressional component into the wave structure in the upper ionosphere (Heilig et al., 2007). Pc3-4 waves demonstrated a localized enhancement of transverse components at midlatitudes attributed to the field line resonance, where the frequency of compressional mode transporting the wave energy from extra-magnetospheric source matches the local frequency of Alfven eigenoscillations (Vellante et al., 2004; Ndiitwani and Sutcliffe, 2009; Heilig et al., 2013). Away from the resonant region, the structure of Pc3-4 waves in the upper ionosphere was dominated by the compressional mode. Moreover, at near-equatorial latitudes the mechanism of Alfven resonance cannot be invoked, because the field lines are totally submerged into the ionospheric plasma and Alfven field line oscillations are strongly damped (Pilipenko et al., 1998). The latitudinal distribution in the top-side ionosphere showed that dayside compressional power in the Pc3 band was most pronounced in two regions: near noon, 09-14 LT at near-equatorial latitudes $\left( \pm 15^{\circ}\right.$ from the geomagnetic equator), and at high latitudes $\sim 75^{\circ}$ (Heilig et al., 2007). However, the overall Pc3 amplitude/phase spatial structure cannot be explained by a simple propagation of fast mode (Matsuoka et al., 1997). Besides resonant conversion into Alfven field line oscillations, the excitation of some cavity mode may be possible.

At near-equatorial latitudes not just a response to ULF wave processes at auroral latitudes is observed, but, a new, not well understood, wave physics can be seen. At nearequatorial stations monochromatic pulsations with elevated amplitudes are often observed (Tanaka et al., 2004). This effect of the "equatorial enhancement of ULF waves" was noticed many decades ago for dayside Pc3-5 waves (Jain and Srinivasacharya, 1975) and nighttime Pi2 pulsations (Osaki et al., 1996; Yumoto, 2001). The equatorial enhancement of ULF waves was $\sim 2-3$ times as compared with more distant stations (Zanandrea et al., 2004). However, a mechanism of such amplification has not been unambiguously identified yet. Possible mechanisms can involve enhancement of the ionospheric currents induced by mid-latitude Alfven wave which spread into the equatorial region with elevated ionospheric Cowling conductance (Shinohara et al., 1997); or direct penetration of compressional wave towards the near-equatorial region without conversion into Alfven field line oscillations (Yumoto and Saito, 1983; Heilig et al., 2007, 2013). Most probably, combination of all these mechanisms takes place, and to resolve them is a challenging goal. A progress in these studies can be achieved only with the comparison of simultaneous observations at low-orbit satellite and ground magnetometers.

Combined satellite-ground observations showed a rather consistent pattern of the compressional mode in the Pi2 and Pc3-4 range transmission from the inner 
magnetosphere through the ionosphere to the ground. However, all these observational results had a qualitative character, and a quantitative comparison with the existing theoretical notions on MHD wave transmission through the ionosphere was made for mid-latitudes only (Pilipenko et al., 2008). In this paper, we estimate the amplitude-phase relationships between the Pi2 and Pc3-4 signals detected simultaneously on CHAMP and low-latitude part of the magnetometer arrays SAMBA and MAGDAS, and compare them with the theoretical model of the compressional mode transmission through the system ionosphere-atmosphere-ground.

\section{The ground and satellite observational facilities}

The CHAMP satellite was launched in 2000 into an almost circular, near polar (inclination $87.3^{\circ}$ ) orbit, with an initial altitude of $454 \mathrm{~km}$ which has changed to $350 \mathrm{~km}$ after 5 years. The satellite data used in this study are the preprocessed fluxgate vector magnetometer data (http://isdc.gfz-potsdam.de/champ/). This instrument samples the magnetic field at a rate of $50 \mathrm{~Hz}$ with a resolution of $0.1 \mathrm{nT}$. In the standard processing the data are averaged to $1-\mathrm{Hz}$ samples.

Data were transformed in a mean field aligned (MFA) coordinate system, where the field model POMME 2.5 (Potsdam Magnetic Model of the Earth) was used as mean field model. This model includes the main field, the field of the ring current, large scale magnetospheric fields, and the crustal anomalies. Components in the orthogonal MFA coordinate system are compressional $\mathrm{B}_{\mathrm{II}}$, poloidal $\mathrm{B}_{\mathrm{P}}$, and toroidal $\mathrm{B}_{\mathrm{T}}$ components, which are respectively parallel to the field line, directed inward, and directed azimuthally eastward. The ULF variations in $\mathrm{B}_{\mathrm{II}}$ component are visually coincident with variations of the total magnetic field B. In the next step POMME 2.5 was subtracted from the measurements. A study of $\mathrm{Pi} 2$ pulsations observed by the CHAMP (Sutcliffe and Lühr, 2003) showed that the correlation between satellite and ground Pi2s can be improved by subtraction of a lithospheric magnetic field anomaly model from the satellite data.

For the monitoring of the ground response to ULF waves, we use the 1-s data from lowest-latitude SAMBA array stations along the Chilean shore (PUT, ANT) and equatorial stations (ANC, GLP) from MAGDAS array. Their codes and coordinates are given in Table 1. Local noon of all stations is $\sim 16: 30 \mathrm{UT}$, so the local time can be estimated as $\mathrm{LT}=\mathrm{UT}-04.30$.
We have examined all CHAMP orbits above the SAMBA/MAGDAS array during June 2003 in search of clear wave signatures. Both ground and satellite magnetometer data were high-pass filtered with the cutoff period $120 \mathrm{~s}$ (for Pi2) and $60 \mathrm{~s}$ (for Pc3).

\section{Examples of typical events}

From the data set of recorded simultaneous signals of $\mathrm{Pc} 3$ and Pi2 types in space and on the ground here we present only typical examples of various classes of ULF events.

\subsection{Night-time low-latitude Pi2 wave trains}

On 2003/06/01, 07:57-08:03 UT $\quad(\mathrm{LT} \sim 03.30) \quad$ Pi2 damping train was detected by CHAMP, when the satellite was at latitude $\Phi \sim 10^{\circ}$ above Central America and approached the dip equator from Northern hemisphere. Pi2 wave is clearly evident at CHAMP in the compressional component with peak-to-peak amplitude $\mathrm{B}_{\|} \sim 0.8 \mathrm{nT}$. On the ground stations the Pi2 amplitude in $\mathrm{X}$-component was $\sim 0.9 \mathrm{nT}$ at low latitude stations (PUT, ANT, GLP) and $\sim 1.8 \mathrm{nT}$ at equatorial stations ANC (Fig. 1).

Cross-spectral analysis (not shown) between CHAMP and PUT records shows that at spectral peak frequency $f \sim 24 \mathrm{mHz}$ the coherence between satellite and ground signals is high, $\gamma \sim 0.95$, whereas the ratio between the satellite and ground spectral amplitudes $G(f) \sim 1.0$. The ground signal is delayed in respect to satellite signal by $\Delta \phi \sim 26^{\circ}$.

\subsection{Nighttime low-latitude Pc3 wave packets}

On 2003/06/08, 06:33-06:39 UT (LT 02) CHAMP detected short-lived oscillations in a nominal Pc3 band (period $\sim 30$ s) (see insert in Fig. 2). The satellite was above Venezuela at $\Phi \sim 14-18^{\circ}$. The signal in space is most visible in compressional component with amplitude $\mathrm{B}_{\|} \sim 0.3 \mathrm{nT}$. On the ground the signal are coherent throughout all stations and has peak-to-peak amplitude in $X$ component $\sim 0.5 \mathrm{nT}$ (PUT, GLP), and $\sim 1.0 \mathrm{nT}$ closer to the equator (ANC) (Fig. 2). The ratio between spectral amplitudes CHAMP/PUT at $f \sim 35 \mathrm{mHz}$ confirms that $G(f) \sim 0.8$. The signals are nearly in-phase: the phase delay of ground signal as compared with signal on the satellite is $\Delta \phi \sim 15^{\circ}$.

During daytime hours no Pi2 signals have been revealed from CHAMP data. Therefore, Pc3 wave signatures only have been analyzed.

Table 1

Low-latitude stations from SAMBA and MAGDAS arrays.

\begin{tabular}{llllccc}
\hline Station name & Station code & Geographic Lat & Geographic Long & CGM Lat & CGM Long & Noon UT \\
\hline Ancon & ANC & -11.79 & -77.16 & 3.1 & 354.4 & $16: 14$ \\
Guadalupe & GLP & -14.06 & -75.95 & -0.06 & 355.6 & $16: 18$ \\
Putre & PUT & -18.33 & -69.50 & -5.5 & 1.4 & $16: 30$ \\
Antofagasta & ANT & -23.39 & -70.24 & -10.3 & 0.7 & $16: 26$ \\
\hline
\end{tabular}




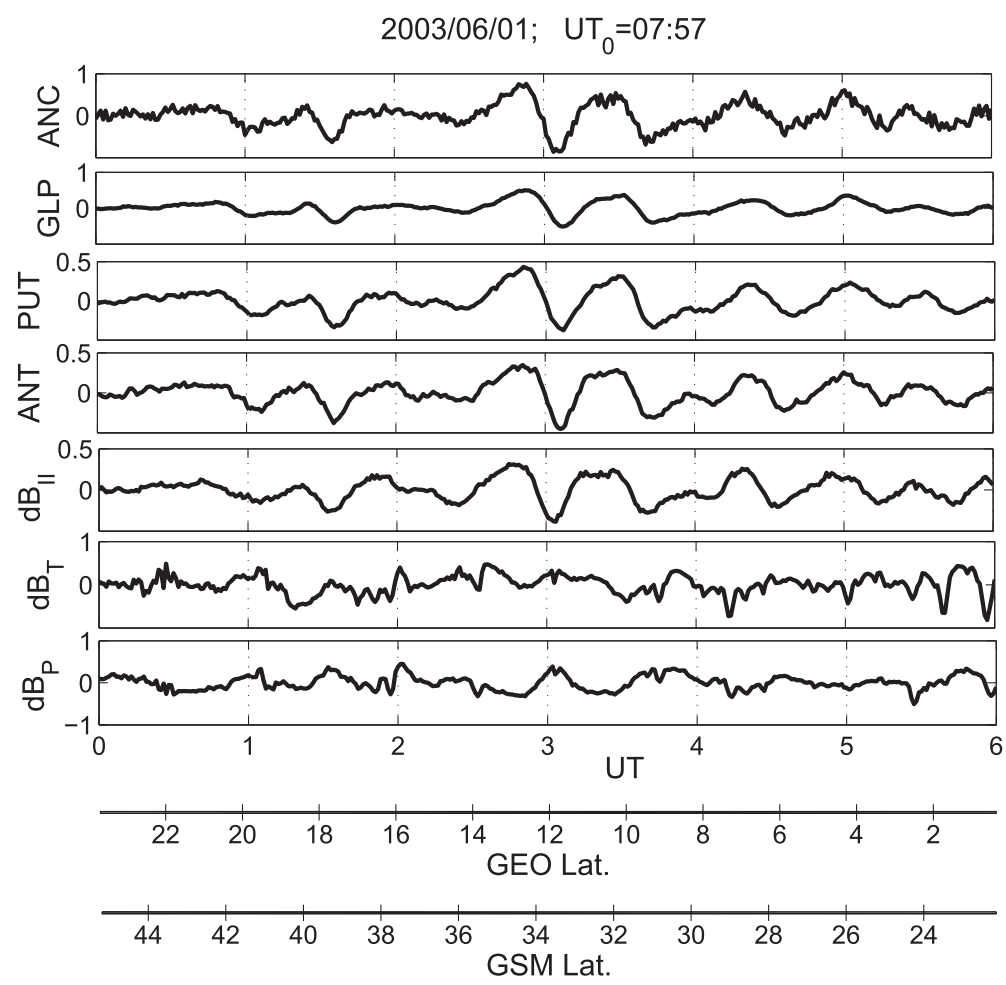

Fig. 1. The low-latitude Pi2 event on 2003/06/01, 07:57-08:03 UT, observed by ground stations ANC, GLP, ANT, PUT ( $X$ component in nT) and CHAMP $\left(\mathrm{B}_{\mathrm{II}}, \mathrm{B}_{\mathrm{T}}\right.$, and $\mathrm{B}_{\mathrm{P}}$ components in $\left.\mathrm{nT}\right)$. The data have been detrended with cut-off period $120 \mathrm{~s}$. The CHAMP geographic and geomagnetic latitudes are shown below time axis.

\subsection{Daytime low-latitude Pc3 waves}

On 2003/06/01, 19:38-19:43 UT (LT 15) Pc3 wave packet was detected by CHAMP above Colombia at the latitude range $\Phi \sim 5^{-} 12^{\circ}$ (insert in Fig. 3). The wave packet was clearly evident at CHAMP in compressional component with amplitude $B_{\|} \sim 0.5 \mathrm{nT}$. On the ground the signal is coherent at all stations with amplitude $X \sim 0.4 \mathrm{nT}$ (Fig. 3), whereas the Pc3 amplitude at equatorial station ANC is nearly 2 times bigger, $X \sim 0.8 \mathrm{nT}$.

Cross-spectral analysis shows that at spectral peak frequency $f \sim 52 \mathrm{mHz}$ the coherence between satellite and ground PUT signals is $\gamma \sim 0.5$, whereas the spectral amplitude ratio CHAMP/PUT is $G(f) \sim 1.4$. However, in contrast to nighttime Pc3 event, the nearly out-of-phase oscillations on the ground and on CHAMP can be seen from a visual inspection. Cross-phase analysis proves that the ground signal is considerably phase-shifted in respect to the satellite signal by $\Delta \phi \sim 120^{\circ}$.

\subsection{Daytime equatorial Pc3 pulsations}

On 2003/06/06, 18:22-18:27 UT (LT 14) Pc3 wave train (insert in Fig. 4) was detected by CHAMP magnetometer when satellite was above Northern Brazil at $\Phi=0-6^{\circ}$. The compressional component has amplitude $B_{\|} \sim 0.4 \mathrm{nT}$. On the ground the signal is evident at low-latitude stations only (GLP, PUT) with amplitude $X \sim 0.2 \mathrm{nT}$ (Fig. 4). At higher latitude (ANT) the signal is lacking, and at equatorial station ANC a strong interference occurred.

Cross-spectral analysis (not shown) gives that at spectral peak frequency range $f \sim 50-60 \mathrm{mHz}$ the coherence between satellite and ground (PUT) signals is $\gamma \sim 0.6$, whereas the spectral amplitude ratio CHAMP/PUT is $G(f) \sim 1.9$. The ground and satellite signals are nearly out-of-phase: the phase shift fluctuates around $\Delta \phi \sim 180^{\circ}$.

\section{Model of MHD wave penetration to the ground at low latitudes}

Both our and previous CHAMP observations have shown that Pc3-4 and Pi2 waves in the upper low-latitude ionosphere are mostly composed from compressional mode. This mode is most easily identified by the disturbed total magnetic field $|\mathrm{B}|$ or field aligned component $B_{\|}$. Therefore, for the interpretation of these coordinated satellite - ground observations we have to consider the fast magnetosonic (FMS) wave interaction with the multi-layered system magnetosphere-thin ionosphere-non-conductive atmosphere-ground. For ULF waves with $T>10 \mathrm{~s}$ the width of the conductive E-layer is much smaller that the wavelength, so the E-layer can be replaced by an infinitely sheet with height-integrated Pedersen and Hall conductances $\Sigma_{\mathrm{P}}$ and $\Sigma_{\mathrm{H}}$, situated at altitude h. We assume that $X$-coordinate corresponds to the North-South (radial) direction, and $Y$ corresponds to the East-West (azimuthal) direction. Magnetic field is inclined by angle $I$ to the 

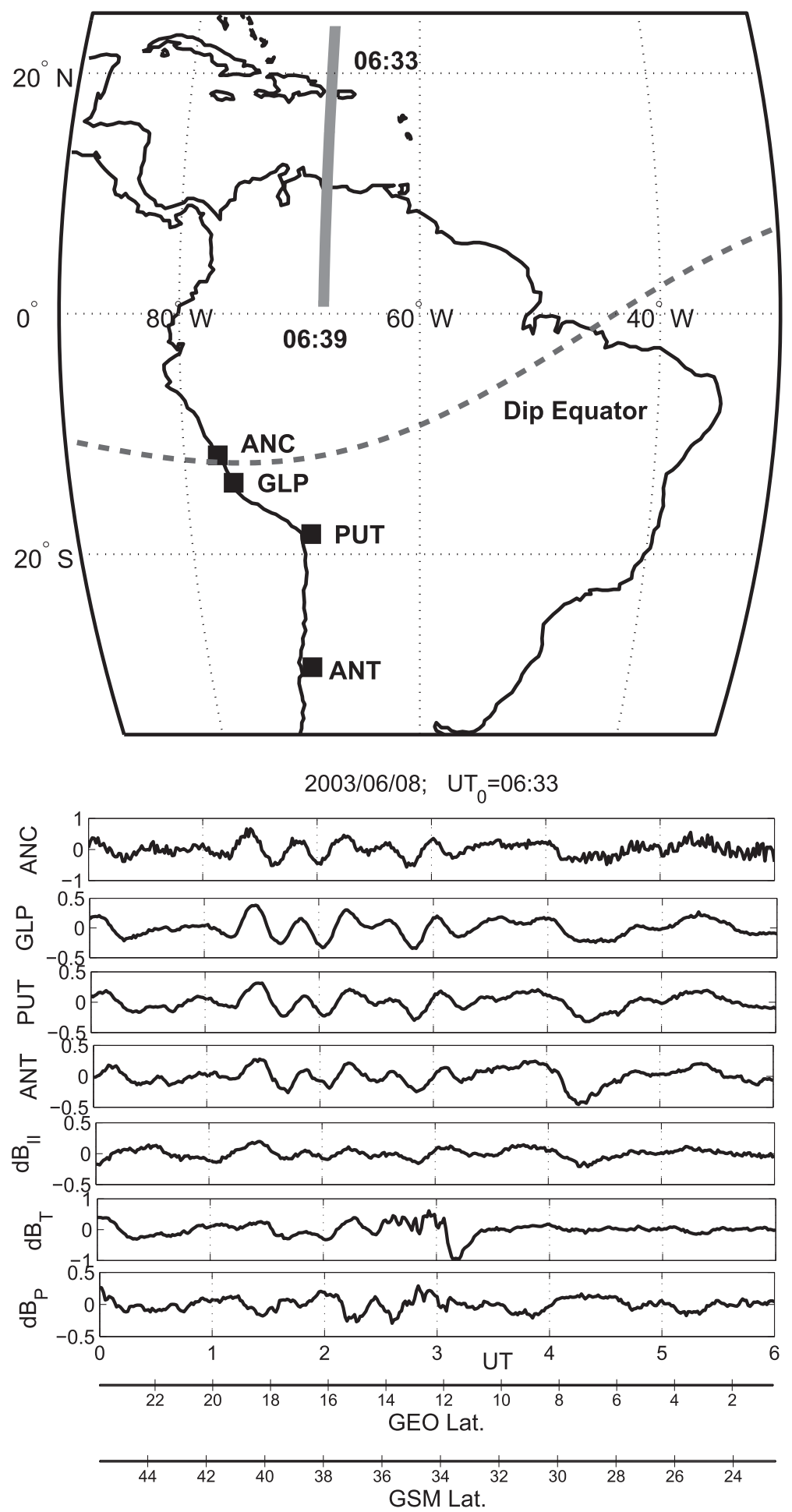

Fig. 2. Night-time low latitude Pc3 event on 2003/06/08, 06.33-06.39 UT. The data have been detrended with cut-off period 120 s. Upper insert: the vertical projection of the CHAMP orbit for this event.

Earth's surface. Here we use some theoretical concepts from the thin sheet approximation (Alperovich and Fedorov, 2007) necessary for the interpretation of the results of simultaneous observations at low-orbiting satellite and ground stations.

The wave scale of the FMS mode in the $\mathrm{Pc} 3 / \mathrm{Pi} 2$ range in the magnetosphere is to be very large (e.g. for $T=30 \mathrm{~s}$, and
Alfven velocity $V_{\mathrm{A}}=10^{3} \mathrm{~km} / \mathrm{s}$, Alfvenic wave number $k_{\mathrm{A}}=\omega / V_{\mathrm{A}} \sim 2 \times 10^{-4} \mathrm{~km}^{-1}$ ). Therefore, FMS waves can hardly reach the bottom ionosphere directly under reasonable horizontal wave numbers, because upon their propagation towards plasma with a higher $\mathrm{V}_{\mathrm{A}}$ they encounter a non-propagation (opaque) region, where the wave becomes evanescent. However, because of its large horizontal scale, 

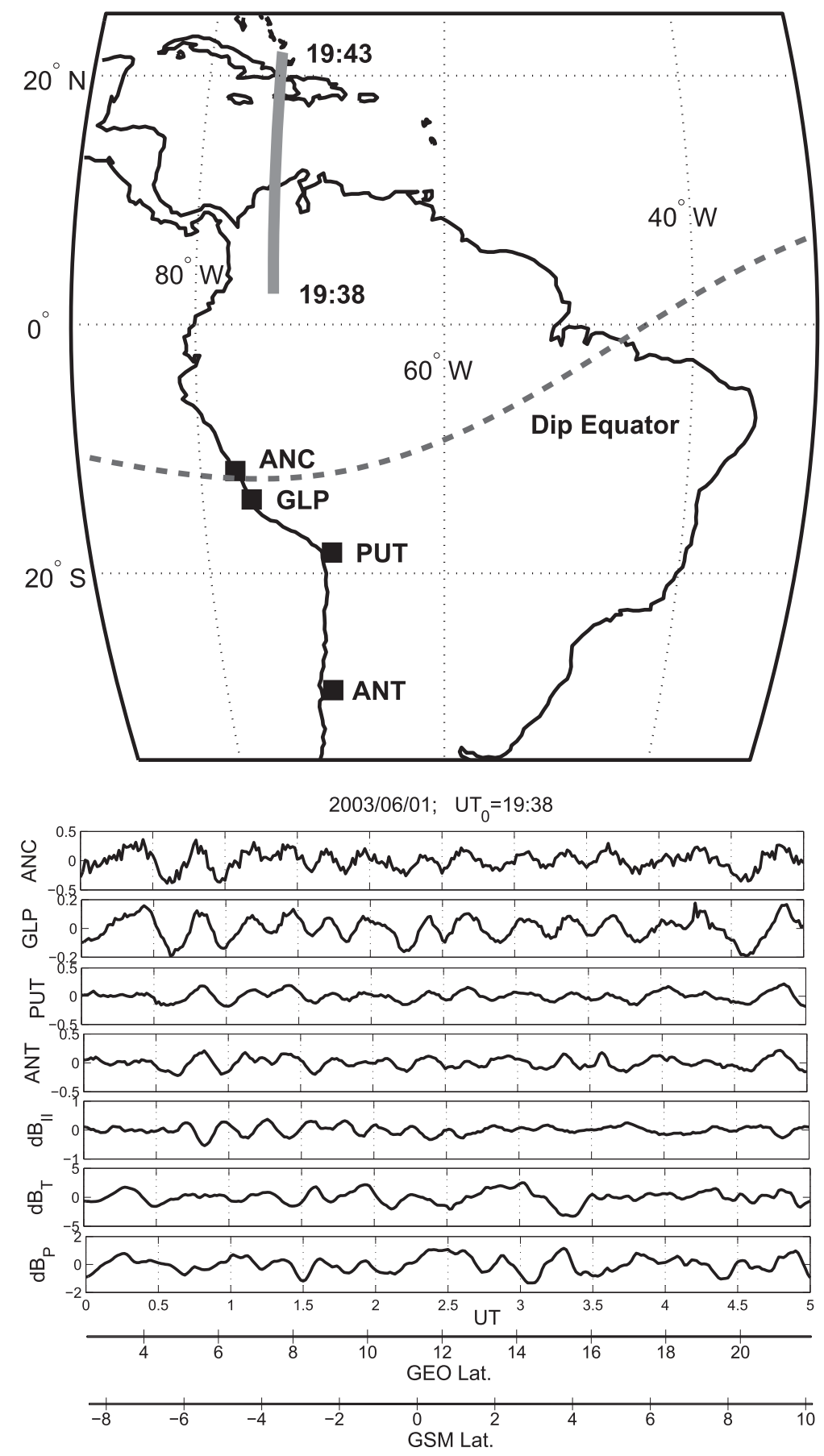

Fig. 3. Daytime low-latitude Pc3 event on 2003/06/01, 19.38-19.43 UT. The same format as in Fig. 1. Upper insert: the vertical projection of the CHAMP orbit for this event.

even an evanescent fast mode can convey significant wave energy towards the Earth. Commonly, the earthward propagation of compressional fast mode was described in the frameworks of 1D (Pekrides et al., 1997; Matsuoka et al., 1997) or 2D (Allan et al., 1996) models. In reality, the spatial distribution of wave power is determined by the diffraction of an incident large-scale FMS mode on a relatively small conductive sphere (Earth). The solution of a similar problem in electrodynamics (Landau and Lifshitz, 1984) prompts that the compressional magnetic field disturbance should decrease away from the dip equator as $B_{\|} \propto \cos \Phi$. This at least partly explains the increase of FMS wave amplitudes upon approaching the geomagnetic equator.

The amplitude ratio and phase delay $\Delta \phi$ between the ground magnetic signal $B_{x}^{(g)}$ and the tangential magnetic component $B_{\tau}$ (at near-equatorial latitudes $B_{\tau} \approx B_{\|}$) of the incident FMS wave is determined by the transmission properties of the whole ionosphere-atmosphere-ground system. The total ULF field in the upper ionosphere is a complicated pattern composed from incident, reflected, 


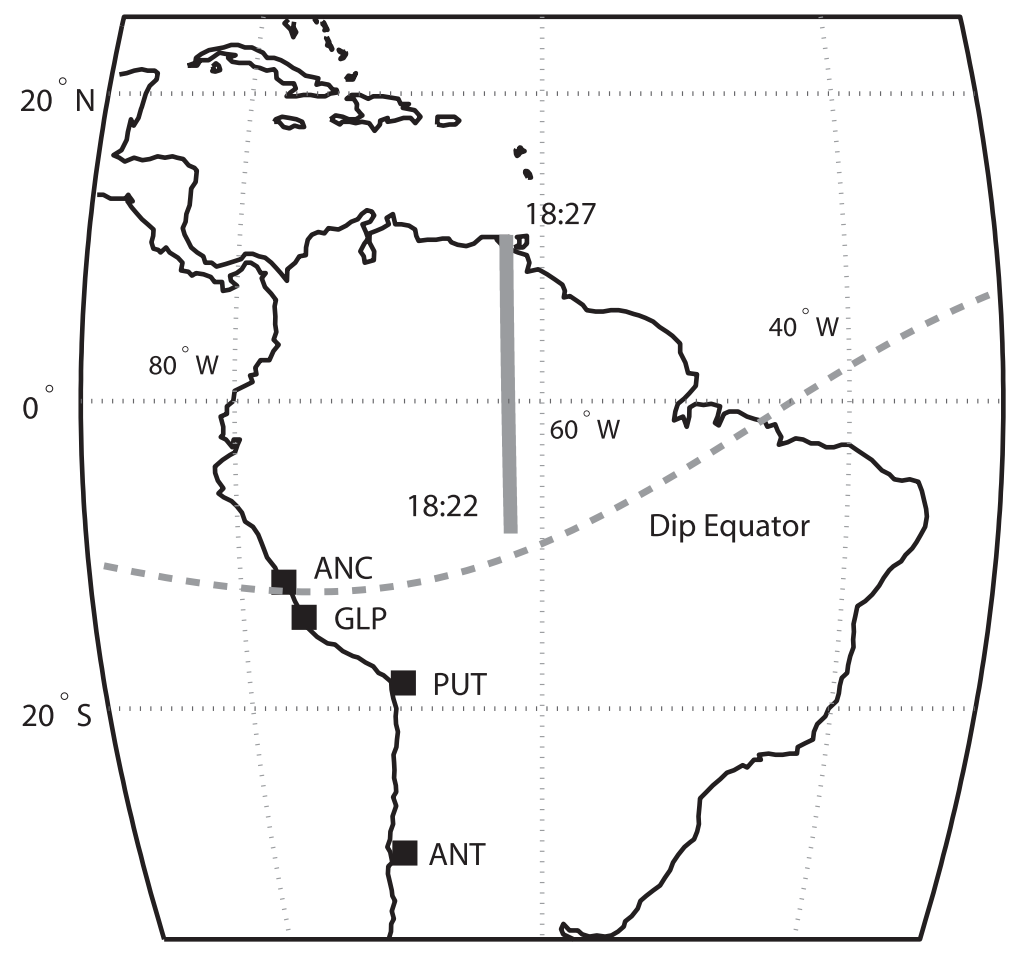

2003/06/06; UT $0=18: 22$

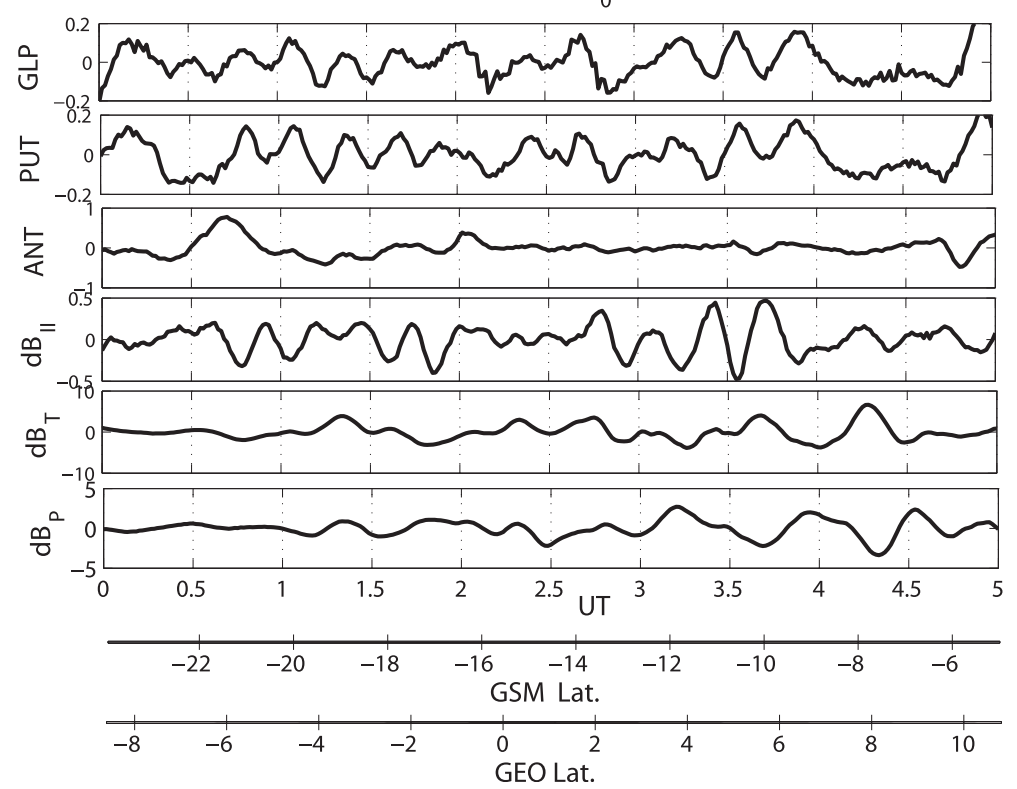

Fig. 4. Daytime equatorial Pc3 event on 2003/06/06, 18.22-18.27 UT, observed by ground stations ANT, PUT, GLP ( $X$ component) and CHAMP. Upper insert: the vertical projection of the CHAMP orbit for this event.

and converted waves. Nonetheless, the thin ionosphere theory (Pilipenko et al., 2008, 2011) provides a remarkably simple relationship between the magnetic disturbance in the ionosphere and its ground response for a highly-conductive ground $\left(\sigma_{g} \rightarrow \infty\right)$, as follows

$\left|\frac{B_{\tau}}{B_{x}^{(g)}}\right|=\sqrt{1+p^{2}}, \quad \Delta \phi=-\tan ^{-1} p, \quad p=\frac{\omega h}{V_{C}}$

Here $V_{C}=\left(\mu_{o} \Sigma_{C}\right)^{-1}$ is the ionospheric Cowling velocity, determined by the Cowling-like combination of the ionospheric conductances $\Sigma_{C}=\Sigma_{P}+\Sigma_{H}^{2} / \Sigma_{P}$. For approximate estimates the relation can be used $V_{C}[\mathrm{~km} / \mathrm{s}] \simeq$ $800 / \sum_{C}[\mathrm{~S}]$. The parameter $p$ controls the penetration of the FMS mode through the ionosphere to the ground. According to (1), the phase delay between satellite and ground must increase with wave frequency $\omega$, as well as with the ionospheric conductance $\Sigma_{C}$.

Let us evaluate on the basis of (1) the transmission of FMS mode with $f=30 \mathrm{mHz}\left(\omega=0.18 \mathrm{~s}^{-1}\right)$ through the thin ionosphere at altitude $h=120 \mathrm{~km}$. Estimate for the 
nightside ionosphere with parameters: $\Sigma_{P}=2 \mathrm{~S}, \Sigma_{H}=2 \Sigma_{P}$, and $\Sigma_{C}=5 \quad \Sigma_{P}=10 \mathrm{~S}$, give $V_{C}=80 \mathrm{~km} / \mathrm{s}$, and $|p| \sim 0.3$. Therefore, the ratio of a signal amplitude in space and on the ground is $\sim 1.04$, whereas the phase shift produced by the ionosphere is $\Delta \phi \sim 16^{\circ}$. Thus, the nightside ionosphere may be considered as nearly transparent for FMS mode, so the incident wave is reflected mainly by Earth's surface.

At dayside at near-equatorial latitudes, assuming that $\Sigma_{P}=20 \mathrm{~S}, \Sigma_{H}=2 \Sigma_{P}$, and $\Sigma_{C}=5 \quad \Sigma_{P}=100 \mathrm{~S}$, one gets $V_{C}=8 \mathrm{~km} / \mathrm{s}$, and $|p|=2.7$. Thus, the dayside ionosphere can partially screen the magnetospheric signal from the ground, $\sim 2.7$ times, and introduce a noticeable phase delay, $\Delta \phi \sim 70^{\circ}$.

\section{Discussion}

Let us compare the observational results from previous publication and presented here with possible scenarios. Many studies already have utilized satellite magnetometer data to demonstrate the compressional mode nature of low-latitude Pi2 pulsations. The analysis of $\mathrm{Pi} 2$ pulsations observed by the AMPTE satellite in the inner magnetosphere and at $L=1.2$ on the ground (Takahashi et al., $1995,1999,2003$ ) found high coherence between satellite and ground pulsations when the satellite was on the nightside and at $L<4$. For these events the magnetic field oscillations at the satellite were dominated by poloidal mode components $B_{\mathrm{P}}$ and $B_{\|}$. The $B_{\|}$component exhibited a cross phase with ground $H$ component of $\Delta \phi \sim 0-90^{\circ}$, whereas a cross phase for $\mathrm{B}_{\mathrm{P}}$ component was $\sim 0^{\circ}$ or $\sim 180^{\circ}$, depending on the location of the satellite in the Northern/Southern hemisphere. This spatial phase structure is consistent with the symmetric field-aligned structure of a compressional cavity mode and standing mode in radial direction with $\mathrm{B}_{\|}$anti-node between reflection points at the equatorial ionosphere and plasmasphere. The mechanism of nighttime Pc3 waves and their correspondence to common daytime Pc3 activity is beyond the scope of this paper and will be considered elsewhere.

Our observations of simultaneous nighttime Pi2 and Pc3 wave packets agree pretty well with the prediction of the model describing the FMS wave interaction with the thin ionosphere, taking into account that satellite observations have not been made perfectly above a station. The considered events are consistent with the observational results of Heilig et al. (2007) for CHAMP, Han et al. (2004) for Orsted, and Takla et al. (2011) for MAGDAS trans-equatorial array, all showing a wide equatorial maximum of compressional Pc3/Pi2 amplitudes. According to the theoretical predictions, typical night side ionosphere should not produce any considerable attenuation of $\mathrm{Pc} 3 / \mathrm{Pi} 2$ wave amplitude, and should introduce just a small phase delay between the ground and space ULF signals. Indeed, the signal at CHAMP is leading the signal on the ground by $\sim 15-25^{\circ}$, whereas the amplitude ratio is about 1 . Thus, the scenario of a direct transmission of the ULF wave energy to the ground at near-equatorial latitudes via compressional mode propagation is well justified by a good agreement between our theoretical model and other satellite/ground observations.

The amplitude and phase changes introduced by the dayside ionosphere on compressional Pc3-4 waves passing through it was also studied by Jadhav et al. (2001), using scalar magnetometer onboard Oersted satellite (altitude $650-900 \mathrm{~km})$ and low-latitude stations. Around $25 \%$ of passes exhibited oscillations in the frequency band 10 $100 \mathrm{mHz}$ in the selected longitude zone. The cross-phase analysis of the satellite and ground oscillations showed that the phase shift was $\Delta \phi \sim 0^{\circ}$ at low frequencies, but it increased with frequency, as well as with the ionospheric conductivity, e.g. $\Delta \phi \sim 0-60^{\circ}$ at $f=20 \mathrm{MHz}$. The features predicted by the theory of the FMS mode interaction with the system ionosphere-ground (1): increase of attenuation and phase delay with wave frequency and ionospheric conductance, corresponds well these Orsted/ground observations of dayside compressional Pc3 waves.

Equatorial electrojet with elevated conductance occupies a narrow equatorial zone $\sim 1-2^{\circ}$. Though the $1 \mathrm{D}$ model presented above cannot be directly applied to the wave transmission through the inhomogeneous ionosphere, one may qualitatively expect that enhanced conductance above the dip equator produces a higher wave absorption as compared with near-by regions. This may qualitatively interpret a highly localized local minimum in latitudinal distribution of Pc3 wave power just above a dip equator found by Takla et al. (2011).

However, some near-equatorial peculiarities of ULF wave structure are not well understood yet. Matsuoka et al. (1997) found a significant phase shift $\sim 150^{\circ}$ of dayside equatorial $(L<1.01) \mathrm{Pc} 3$ pulsations as compared with those at low-latitude stations. Our ground observations have revealed equatorial enhancement of amplitude $\sim 2$ times, but no phase peculiarities at equatorial MAGDAS stations.

The nearly out-of-phase relationship $\left(\sim 120^{\circ}\right)$ of dayside Pc3 pulsations waves detected simultaneously on CHAMP and on the ground SAMBA/MAGDAS stations are not unique. Similar nearly out-of phase relationships can be seen in the magnetograms of dayside Pc3 waves presented in (Heilig et al., 2007). The cross-phase between compressional Pc3 waves detected by Oersted and ground stations (Jadhav et al., 2001) in fact varied in a wide range: for morning events it tends to concentrate around $60-70^{\circ}$, but for near-noon events it reached $\sim 150^{\circ}$. The theory of FMS wave interaction with the ionosphere is in a reasonable agreement with the observed attenuation $\sim 1.5-2$ times between low-Earth orbit and the ground, but under realistic parameters large phase delays $\sim 120-180^{\circ}$ cannot be produced by a magnetospheric compressional wave leaking through the ionosphere.

One possibility may be related to the Pc3 wave transmission to near-equatorial latitudes via oscillating ionospheric currents, spreading from a higher latitude Alfvenic oscillations. Indeed, oscillating E-W ionospheric current in 
E-layer produces out-of-phase magnetic disturbances in $\mathrm{N}-\mathrm{S}$ components on the ground and nearly along the geomagnetic field above the E-layer. Probably, in realistic situations, combination of both mechanisms takes place, so a more advanced theoretical model is needed, which would consider the incidence of mixed Alfven and FMS modes.

\section{Conclusion}

The mechanism of near-equatorial enhancement of Pc3/ $\mathrm{Pi} 2$ waves could be related either to the equatorial enhancement of oscillating ionospheric currents due to the elevated Cowling conductance of the near-equatorial ionosphere, or be a result of direct compressional mode transmission to the ground. The simultaneous CHAMP and ground observations helped to resolve this ambiguity. The nighttime Pi2 and Pc3 waves at near-equatorial latitudes are produced by the direct transmission of the compressional fast mode towards the ground. The observed amplitude-phase relationships between the nightside ionospheric and ground signals match well predictions of the thin ionosphere model. However, satellite/ground amplitude/phase relationships of low-latitude dayside Pc3 waves are not consistent with only one or another mechanism.

\section{Acknowledgments}

The useful discussions with E. Fedorov and N. Yagova are appreciated. This study is partly supported by the Grant No. 13-05-90436 from RFBR (VP). We acknowledge the SAMBA data provided by E. Zesta, and data from ANC station provided by T. Uozumi and J. Ishitsuka. We appreciate valuable comments of both Reviewers.

\section{References}

Allan, W., Menk, F.W., Fraser, B.J., Li, Y., White, S.P., 1996. Are lowlatitude Pi2 pulsations cavity/waveguide modes? Geophys. Res. Lett. 23, 765-763.

Alperovich, L.S., Fedorov E.N., 2007. Hydromagnetic Waves in the Magnetosphere and the Ionosphere. Astrophysics and Space Science Library, vol. 353, 2009, XXIV, 418p. ISBN:978-1-4020-6636-8.

Han, D.-S., Iyemori, T., Nosé, M., McCreadie, H., Gao, Y., Yang, F., Yamashita, S., Stauning, P., 2004. A comparative analysis of low latitude Pi2 pulsations observed by Ørsted and ground stations. J. Geophys. Res. 109, A10209. http://dx.doi.org/10.1029/2004JA010576.

Heilig, B., Lühr, H., Rother, M., 2007. Comprehensive study of ULF upstream waves observed in the topside ionosphere by CHAMP and on the ground. Ann. Geophys. 25, 737-754.

Heilig, B., Sutcliffe, P.R., Ndiitwani, D.C., Collier, A.B., 2013. Statistical study of geomagnetic field line resonances observed by CHAMP and on the ground. J. Geophys. Res. 118, 1934-1947. http://dx.doi.org/ $10.1002 /$ jgra. 50215 .

Jadhav, G., Rajaram, M., Rajaram, R., 2001. Modification of daytime compressional waves by the ionosphere: first results from Oersted. Geophys. Res. Lett. 28, 103-106.

Jain, A.R., Srinivasacharya, K.G.A., 1975. Study of magnetic pulsations in the Indian equatorial region. J. Atmos. Terr. Phys. 37, 1477-1483.

Kepko, L., Kivelson, M., 1999. Generation of Pi2 pulsation by bursty bulk flows. J. Geophys. Res. 104, 25021-25034.
Keiling, A., Takahashi, K., 2011. Review of Pi2 models. Space Sci. Rev. 161, 63-148.

Kim, K.-H., Takahashi, K., 1999. Statistical analysis of compressional Pc3-4 pulsations observed by AMPTE CCE at $L=2-3$ in the dayside magnetosphere. J. Geophys. Res. 104, 4539-4558.

Kim, K.-H., Takahashi, K., Lee, D.H., Sutcliffe, P.R., Yumoto, K., 2005. Pi2 pulsations associated with poleward boundary intensifications during the absence of substorms. J. Geophys. Res. 110, A01217. http:// dx.doi.org/10.1029/2004JA010780.

Landau, L.D., Lifshitz, E.M., Pitaevskii, L.P., 1984. Electrodynamics of Continuous Media (Course of Theoretical Physics, v. 8). ButterworthHeinemann.

Li, Y., Fraser, B.J., Menk, F.W., Webster, D.J., Yumoto, K., 1998. Properties and sources of low and very low latitude Pi2 pulsations. J. Geophys. Res. 103, 2343-2358.

Matsuoka, H., Takahashi, K., Kokubun, S., Yumoto, K., Yamamoto, T., Solovyev, S.I., Vershinin, E.F., 1997. Phase and amplitude structure of Pc3 magnetic pulsations as determined from multipoint observations. J. Geophys. Res. 102, 2391-2403.

Ndiitwani, D.C., Sutcliffe, P.R., 2009. The structure of low-latitude Pc3 pulsations observed by CHAMP and on the ground. Ann. Geophys. $27,1267-1277$.

Nosé, M., Liou, K., Sutcliffe, P.R., 2006. Longitudinal dependence of characteristics of low-latitude Pi2 pulsations observed at Kakioka and Hermanus. Earth Planet. Space 58, 775-783.

Osaki, H., Yumoto, K., Fukao, K., Shiokawa, K., Menk, F.W., Fraser, B.J., $10 \mathrm{MM}$ observation group, 1996. Characteristics of low-latitude Pi2 pulsations along the $210^{\circ}$ magnetic meridian. J. Geomag. Geoelectr. 48, 1421-1430.

Pekrides, H., Walker, A.D.M., Sutcliffe, P.R., 1997. Global modeling of Pi2 pulsations. J. Geophys. Res. 102, 14343-14354.

Pilipenko, V.A., Yumoto, K., Fedorov, E., Kurneva, N., Menk, F., 1998. Field line Alfven oscillations at low latitudes. Mem. Fac. Sci. Kyushu Univ. Ser. D Earth Planet. Sci. XXX (1), 23-43.

Pilipenko, V., Fedorov, E., Heilig, B., Engebretson, M.J., 2008. Structure of ULF Pc3 waves at low altitudes. J. Geophys. Res. 113, A11208.

Pilipenko V., Fedorov, E., Heilig, B., Engebretson, M.J., Sutcliffe, P., Luehr, H., 2011. ULF waves in the topside ionosphere: satellite observations and modeling. In: The Dynamic Magnetosphere, IAGA Special Sopron Book Series, vol. 3, Springer Science+Business Media (Chapter 14).

Shinohara, M., Yumoto, K., Yoshikawa, A., Saka, O., Solovyev, S.I., Vershinin, E.F., Trivedi, N.B., 210 MM magnetic observation group, 1997. Wave characteristics of daytime and nighttime Pi2 pulsations at the equatorial and low latitudes. Geophys. Res. Lett. 24, 2279-2282.

Sutcliffe, P.R., Lühr, H., 2010. A search for dayside geomagnetic Pi2 pulsations in the CHAMP low-Earth-orbit data. J. Geophys. Res. 115, A05205. http://dx.doi.org/10.1029/2009JA014757.

Sutcliffe, P.R., Lühr, H., 2003. A comparison of Pi2 pulsations observed by CHAMP in low Earth orbit and on the ground at low latitudes. Geophys. Res. Lett. 30, 2105 . http://dx.doi.org/10.1029/ 2003GL018270.

Sutcliffe, P.R., Lyons, L.R., 2002. Association between quiet-time Pi2 pulsations, poleward boundary intensifications, and plasma sheet particle fluxes. Geophys. Res. Lett. 29, 1293. http://dx.doi.org/ 10.1029/2001GL014430.

Sutcliffe, P.R., Yumoto, K., 1991. On the cavity mode nature of low latitude Pi2 pulsations. J. Geophys. Res. 96, 1543-1551. http:// dx.doi.org/10.1029/90JA02007.

Takahashi, K., Ohtani, S.-I., Anderson, B.J., 1995. Statistical analysis of Pi2 pulsation observed by the AMPTE CCE spacecraft in the inner magnetosphere. J. Geophys. Res. 100, 21929-21941.

Takahashi, K., Anderson, B.J., Yumoto, K., 1999. Upper atmosphere research satellite observation of a Pi2 pulsation. J. Geophys. Res. 104, 25035-25045.

Takahashi, K., Lee, D.-H., Nosé, M., Anderson, R.R., Hughes, W.J., 2003. CRRES electric field study of the radial mode structure of Pi2 
pulsations. J. Geophys. Res. 108, 1210. http://dx.doi.org/10.1029/ 2002JA009761.

Takahashi, K., Kokubun, S., Matsuoka, H., et al., 1994. Geotail observation of magnetosonic Pc3 waves in the dayside magnetosphere. Geophys. Res. Lett. 21, 2899-2902.

Takla, E.M., Yumoto, K., Cardinal, M.G., et al., 2011. A study of latitudinal dependence of Pc 3-4 amplitudes at 960 magnetic meridian stations in Africa. Sun Geosphere 6 (2), 65-70.

Tanaka, Y.-M., Yumoto, K., Yoshikawa, A., Shinohara, M., Kawano, H., Kitamura, T.-I., 2004. Longitudinal structure of Pc3 pulsations on the ground near the magnetic equator. J. Geophys. Res. 109 (A03), 201. http://dx.doi.org/10.1029/2003JA009903.

Teramoto, M., Nosé, M., Sutcliffe, P.R., 2008. Statistical analysis of Pi2 pulsations inside and outside the plasmasphere observed by the polar orbiting DE-1 satellite. J. Geophys. Res. 113, A07203. http:// dx.doi.org/10.1029/2007JA012740.

Teramoto, M., Takahashi, K., Nosé, M., Lee, D.-H., Sutcliffe, P.R., 2011. $\mathrm{Pi} 2$ pulsations in the inner magnetosphere simultaneously observed by the AMPTE/CCE and DE-1 satellites. J. Geophys. Res. 116, A07225. http://dx.doi.org/10.1029/2010JA016199.

Vellante, M., Lühr, H., Zhang, T., Wesztergom, V., Villante, U., Lauretis, M., Piancatelli, A., Rother, M., Schwiegenschuh, K., Koren, W.,
Magnes, 2004. Ground/satellite signatures of field line resonance: a test of theoretical predictions. J. Geophys. Res. 109, A06210. http:// dx.doi.org/10.1029/2004JA010392.

Yeoman, T.K., Orr, D., 1989. Phase and spectral power of mid-latitude Pi2 pulsations: evidence for a plasmaspheric cavity resonance. Planet. Space Sci. 37, 1367-1383.

Yumoto, K., Takahashi, K., Sakurai, T., Sutcliffe, P.R., Kokubun, S., Luhr, H., Saito, T., Kuwashima, M., Sato, N., 1990. Multiple groundbased and satellite observations of global Pi2 magnetic pulsations. J. Geophys. Res. 95, 15175-15184.

Yumoto, K., CPMN Group, 2001. Characteristics of Pi2 magnetic pulsations observed at the CPMN stations: a review of the STEP results. Earth Planets Space 53, 981-992.

Yumoto, K., Saito, T., 1983. Relation of compressional HM waves at GOES-2 to low-latitude Pc3 magnetic pulsations. J. Geophys. Res. 88, 10041

Zanandrea, A., Da Costa, J.M., Dutra, S.L.G., Trivedi, N.B., Kitamura, T., Yumoto, K., Tachihara, H., Shinohara, M., Saotome, O., 2004. Pc3-4 geomagnetic pulsations at very low latitude in Brazil. Planet. Space Sci. 52, 1209-1215. 